\begin{tabular}{cc} 
Journal of Environmental \\
Analysis and Progress \\
ISSN: $2525-815 \mathrm{X}$ & Journal homepage: $\underline{\text { www.jeap.ufrpe.br/ }}$ \\
\hline
\end{tabular}

\title{
Pegada hídrica em indústria de beneficiamento de jeans no Agreste Pernambucano
}

\section{Water footprint of the jeans processing industry in the Agreste of Pernambuco}

\author{
Mirella Alexandre Viana ${ }^{\mathrm{a}}$, Elizabete Buonora de Souza Lira ${ }^{\mathrm{a}}$, Carla de Paula Gomes Coelho ${ }^{\mathrm{a}}$, Ana \\ Paula Xavier de Gondra Bezerra ${ }^{\mathrm{a}}$, Emmanuelle Maria Gonçalves Lorena ${ }^{\mathrm{a}}$, Romildo Morant de \\ Holanda $^{\mathrm{a}}$
}

${ }^{a}$ Universidade Federal Rural de Pernambuco-UFRPE. Rua Dom Manoel de Medeiros, s/n, Dois Irmãos, Recife-PE, Brasil. CEP: 52171-900. E-mail: mirellaviana@hotmail.com, elizabete.buonora@hotmail.com, carlapgcoelho@ hotmail.com, romildomorant@gmail.com.

A R T I C L E I N F O

Recebido 27 Nov 2017

Aceito 28 Dez 2017

Publicado 22 Jan 2018

\begin{abstract}
A B S T R A C T
The textile processing industry is a sector that presents high polluting potential, because its processes demands large volumes of water and generate chemically complex effluents. In response to a need to implement techniques and tools to manage the sustainable use of water, the concept of Water Footprint (WF) was created. It consists in calculating the total volume of water consumed directly and indirectly by humans and in the production processes. Because it is an issue that has not yet been well discussed, the objective of this study was to evaluate the Blue Water Footprint of the denim dyeing process in four textile laundries located at the Pernambuco textile industry (complex). A telephone interview was conducted with four companies (A, B, C and D) of jeans processing located in the city of Caruaru, Pernambuco. Considering the data obtained, it was possible to estimate the water footprint of the process for each studied company, where it presented the following results: $17.74,24,14.93$, 60 litres per piece. It was concluded that having the knowledge of the water footprint of a textile dyeing process demonstrates that this activity is characterized as having significant potential for pollution due to the high volume of water required for its production process and that the reuse and correct management of the waste water can work as mitigating alternatives.
\end{abstract}

Keywords: Water resources, textile dyeing process, water footprint.

\section{R E S U M O}

A indústria de beneficiamento têxtil é um setor que apresenta elevado potencial poluidor, pois em seus processos demandam grandes volumes de água e geram efluentes quimicamente complexos. Em resposta à necessidade de implementar técnicas e ferramentas voltadas ao gerenciamento do uso sustentável da água foi criado o conceito Pegada Hídrica (PH), que consiste no cálculo do volume total de água consumida de forma direta e indireta pelos seres humanos e nos processos de produção. Por ser um tema ainda pouco discutido, o objetivo desse estudo consistiu em avaliar a Pegada Hídrica Azul do processo de beneficiamento do jeans em quatro lavandarias têxtil localizadas no Pólo de Confecções do Agreste de Pernambuco. Foi realizada entrevista telefônica com quatro empresas (A, B, C e D) de beneficiamento de jeans localizadas na cidade de Caruaru, Pernambuco. Considerando os dados obtidos, foi possível realizar uma estimativa da pegada hídrica do processo para cada empresa estudada, onde as mesmas apresentaram $\mathrm{PH}$ de $17.74,24,14.93$ e 60 litros por peças, respectivamente. Concluiu-se que o conhecimento da pegada hídrica do processo de beneficiamento têxtil mostrou que essa atividade é caracterizada como de significativo potencial poluidor devido ao elevado volume de água necessário para o seu processo produtivo e que o reuso e o 
correto manejo das águas residuais podem funcionar como alternativas mitigadoras.

Palavras-Chave: Recursos hídricos, beneficiamento têxtil, pegada hídrica.

\section{Introdução}

A água, apesar de ser um recurso natural abundante no planeta, ocupando, aproximadamente, $70 \%$ da superfície (FAO, 2007), tornou-se uma condicionante no crescimento mundial (OCDE, 2015). A baixa disponibilidade de água doce, o correspondente a apenas 2,5\% desse volume, se reflete no problema da escassez hídrica, que ocorre quando a oferta desse recurso em uma região é inferior à sua demanda (FAO, 2007).

Quando um país dispõe de menos de $1.000 \mathrm{~m}^{3} /$ pessoa/ano de água fica caracterizada a escassez hídrica, caso esta disponibilidade seja entre 1.000 e $1.700 \mathrm{~m}^{3}$.pessoa ${ }^{-1} \mathrm{ano}^{-1}$ fica indicada uma "tensão hídrica" (OPAS/CEPIS, 2002).

Conforme estudos das Organizações das Nações Unidas (ONU), o problema da escassez hídrica é encontrado em grande parte dos países. Estima-se que a condição de escassez absoluta de água estará atingindo cerca de 1.800 milhões de pessoas até o ano 2025 (UN-WATER, 2006).

O Brasil, apesar de ser um país que possui grandes reservas hídricas, possui regiões com baixa disponibilidade de água doce, pois fatores como a elevada extensão territorial, as condições climáticas e a diversificada configuração geográfica exercem influência direta na distribuição da disponibilidade hídrica. No território do país passam cerca de $260.000 \mathrm{~m}^{3} \cdot \mathrm{s}^{-1}$ de água, porém a bacia do Rio Amazonas concentra grande parte dessa vazão (cerca de $\left.205.000 \mathrm{~m}^{3} \cdot \mathrm{s}^{-1}\right)$, restando para as bacias das demais regiões apenas $55.000 \mathrm{~m}^{3} \cdot \mathrm{s}^{-1}$ de vazão média (ANA, 2016).

$\mathrm{Na}$ região Nordeste, além da reduzida concentração dos recursos hídricos, correspondente a apenas 3,3\% do país (IBGE/ANA, 2010), aspectos hidrogeológicos associados aos reduzidos índices pluviométricos e aos elevados índices de evapotranspiração, decorrentes das elevadas temperaturas e baixas precipitação e umidade do solo, resultam em baixos valores de disponibilidade hídrica para essa região (ANA, 2016).

A redução da disponibilidade de água possui como principais fatores o crescimento populacional, a mudança global do clima e o aumento da demanda de água decorrente da mudança de estilo de vida das pessoas (Peters, 2006).

O lançamento de efluentes domésticos ou industriais sem o tratamento adequado nos corpos receptores afeta diretamente a disponibilidade de água doce, pois resulta na sua poluição, podendo ser capaz de afetar a qualidade da água ao ponto de impedir o seu uso para diversas atividades, principalmente para consumo humano (UNESCO, 2017).

Segundo dados do Ministério do Meio Ambiente, as indústrias: química, têxtil, de pesticida, tinta, medicamento, papel e celulose são os setores que apresentam significativo potencial poluidor (MMA, 2013).

Nesse contexto, a indústria têxtil é bastante relevante, pois é um dos mais importantes segmentos de transformação industriais do Brasil e do mundo (SEBRAE, 2000).

As lavandarias de jeans estão entre as atividades industriais mais impactantes na área ambiental de devido ao seu elevado potencial poluidor e complexidade química dos efluentes gerados. $\mathrm{O}$ processo produtivo da indústria de beneficiamento têxtil tem como principal insumo a água (Allègre et al., 2006), a perda de água durante o processo industrial nas lavandarias pode chegar a $10,6 \%$, tendo como principais causas os vazamentos nas tubulações, as máquinas com problemas e a evaporação (Alkaya \& Demirer, 2014)

A fim de combater os problemas de disponibilidade e conservação dos recursos naturais, está ocorrendo um processo de mudança de comportamento, e as empresas, seja por força de lei ou por conscientização, começam a adotar uma postura sustentável (Donaire, 2011).

Diante do crescente problema da crise hídrica, torna-se fundamental a utilização de ferramentas de gestão ambiental que buscam auxiliar no uso dos recursos naturais de forma sustentável. Considerando algumas metodologias aplicadas para definição de indicadores de sustentabilidade ambiental pode-se contemplar para externar o uso sustentável dos recursos hídricos, a utilização de ferramentas como a Família das Pegadas, a pegada hídrica, pegada ecológica e pegada de carbono como uma iniciativa nos estudos ambientais (Silva et al., 2015).

De acordo com Galli et al. (2012), as Pegadas Ecológicas do carbono e hídrica devem ser consideradas como ferramentas capazes de identificar as pressões antrópicas em várias repartições da biosfera, atmosfera e hidrosfera.

A utilização da Pegada Hídrica como indicador abrangente da apropriação de recursos hídricos, incluindo usos diretos e indiretos, ao longo das cadeias produtivas, vem sendo integrado ao meio científico e empresarial de 
forma positiva, porém ainda não existem muitos estudos sobre este tema no Brasil (Bleininger \& Kotsuka, 2017).

A pegada hídrica é composta por três componentes: Pegada Hídrica Azul, Pegada Hídrica Verde e Pegada Hídrica Cinza.

A pegada azul é um indicador do consumo da água doce superficial e subterrânea de uma bacia hidrográfica (Ercinet et al., 2013; Ercine \& Hoestra, 2014). Essa pegada representa a perda dessas águas ao longo de uma cadeia produtiva, seja pela incorporação ao produto (consumo), por evaporação, ou pelo retorno à outra bacia hidrográfica ou ao mar.

A pegada verde trata do volume de água proveniente da precipitação continental que é utilizado no processo produtivo, e que não sofre escoamento superficial ou infiltração, logo, que não contribui para a reposição das águas subterrâneas, ou seja, é um indicador da quantidade de água verde consumida (GerbensLeenes et al., 2012), sendo bastante relevante para a produção agrícola (Bosire et al., 2015; Ercin et al., 2013).

Quanto à Pegada Hídrica Cinza, ela refere-se ao volume de água necessário para diluir os poluentes a fim de que passem a atender os padrões de qualidade estabelecidos pelas legislações ambientais vigentes para o lançamento em corpos receptores (Hoekstra et al., 2011).

Os estudos de pegada hídrica podem ser aplicados em diversos contextos e ter várias finalidades, como as pegadas hídricas de um processo, de um produto, de um consumidor, de um grupo de consumidores, de uma área delimitada geograficamente, de um negócio, de um setor de negócios ou da humanidade como um todo (Hoekstra et al., 2011).

De acordo com a finalidade a que se a propõe, análise da pegada hídrica é realizada de forma diferente. No que se refere à pegada hídrica de um processo, ela representa o volume de água por unidade de produto (Hoekstra et al., 2011).

Desta forma, considerando que o conceito de Pegada Hídrica é um assunto pouco discutido em algumas atividades e que as indústrias de beneficiamento têxtil consomem elevados volumes de água, o objetivo desse estudo consistiu em realizar a avaliação da Pegada Hídrica Azul do processo produtivo das lavandarias Têxteis do Pólo de Confecções do Agreste de Pernambuco.

\section{Material e Métodos}

Conforme Amaral et al. (2012), na Região Nordeste, mais especificadamente no Agreste Pernambucano, o desenvolvimento e expansão das indústrias de confecção e têxtil tem adquirido relevância, sendo atualmente essa a principal atividade industrial da Mesorregião.

No agreste de Pernambuco se encontra em pleno desenvolvimento econômico, sendo observado um grande número de indústrias têxteis por toda região. O Arranjo Produtivo Local (APL) da confecção do agreste pernambucano, como é conhecido, é um importante pólo de confecções de tecido do agreste especializado em "jeans", onde estão inseridos quatro municípios, dentre os 16 que compõe o agreste do estado de Pernambuco: Caruaru, Santa Cruz do Capibaribe, Toritama e Riacho das Almas (Figura 1) (Marcelino, 2013).

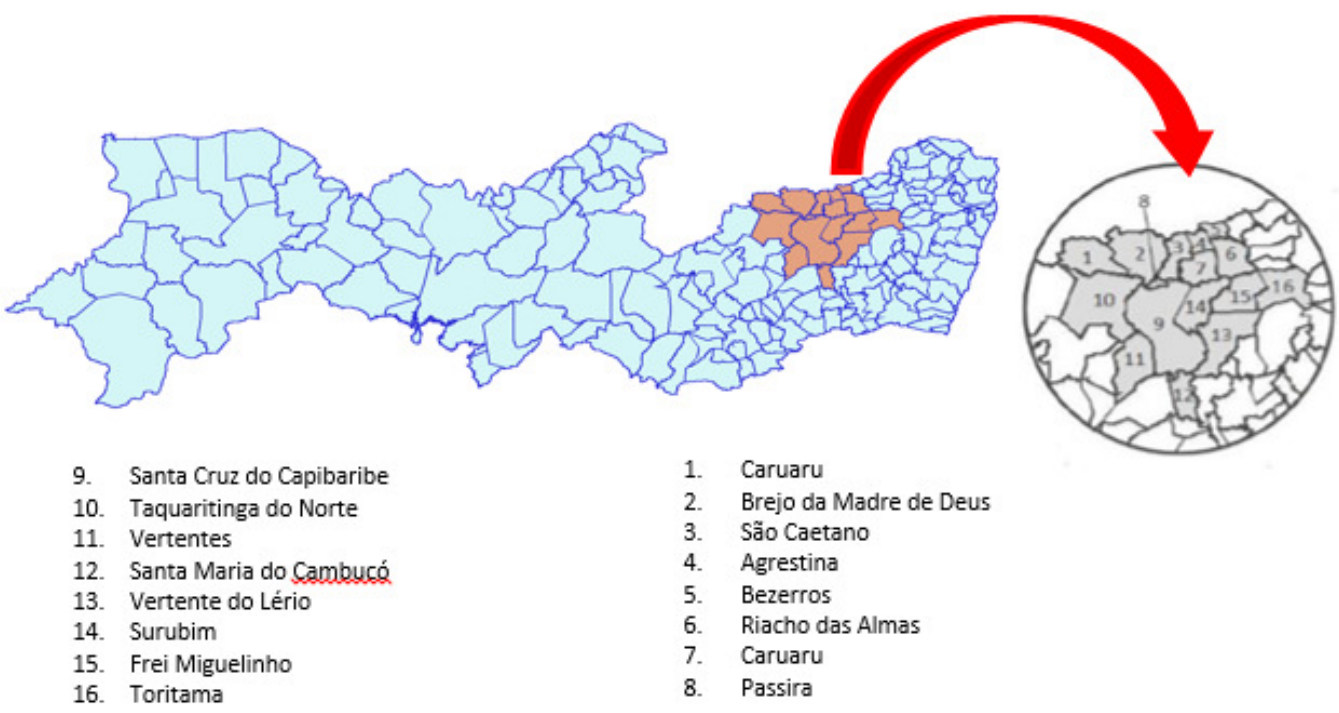

Figura 1. Localização das cidades do pólo têxtil pernambucano. Fonte: Adaptado de Trindade et al. (2013). 
Pernambuco possui o terceiro maior setor de confecções do Brasil, ficando atrás apenas do Rio de Janeiro e São Paulo, com faturamento representando 7\% do PIB pernambucano (Melo, 2012). A região agreste concentra $60 \%$ da produção do estado, onde existem 12 mil empreendimentos pertencentes ao Arranjo Produtivo Local (APL) de confecções, concentrados principalmente nos municípios de Caruaru, Santa Cruz do Capibaribe, Toritama e Surubim. O complexo emprega cerca de 76 mil pessoas, sendo os principais produtos fabricados: o jeans, a malharia em algodão e as peças de moda casual em tecido (SEBRAE, 2008 apud Melo, 2012).

O Pólo de Confecção do Agreste (PCA) é um dos pólos de desenvolvimento da economia do estado de Pernambuco estruturado como um Arranjo Produtivo Local (APL) devido às características específicas dos municípios que os compõem. Caruaru, Toritama e Santa Cruz do Capibaribe são cidades que geograficamente estão muito próximas, seus produtos se assemelham; peças do vestuário destinadas ao mercado de moda popular de vários estados do Brasil (Barros, 2009). O mesmo autor afirma que no Estudo de Caracterização Econômica do Pólo de Confecções do Agreste Pernambucano desenvolvido pela FADE para o SEBRAE aponta que são produzidas 57,8 milhões de peças por mês, nos municípios do Pólo.

\section{Aplicação da ferramenta pegada hídrica}

A metodologia da Pegada Hídrica mede a utilização de água nas atividades humanas e na produção de bens e serviços, considerando o consumo de água e sua contaminação. Trata-se de um instrumento de gestão que se baseia na promoção da eficiência no uso da água, na busca do uso sustentável da água e no estímulo ao compartilhamento equitativo da limitada disponibilidade hídrica (Teixeira, 2016).

Visando realizar a avaliação da pegada hídrica azul ao processo de beneficiamento jeans em uma lavandaria industriais do Pólo Têxtil do agreste de Pernambuco, a metodologia utilizada consistiu no levantamento de dados e informações disponíveis na literatura especializada, com o objetivo de aplicar essa ferramenta de gestão ambiental de acordo com metodologia citada por Hoekstra et al. (2011), no Manual da Pegada Hídrica, criado pelo Water Footprint Network (WFN).

Conforme citado no Manual da Pegada Hídrica, a pegada hídrica de um processo é expressa como o volume de água por unidade de tempo. Quando dividida pela quantidade de produtos que resultam do processo (unidades de produto por unidade de tempo), pode também ser expressa como o volume de água por unidade de produto, segundo a Equação 1 (Hoekstra et al., 2011).

$$
\mathrm{PH}_{\text {proc }}[\mathrm{p}]=\frac{\sum_{s=1}^{n} \text { PHproc }[\mathrm{S}]}{\mathrm{P}[\mathrm{p}]}
$$

onde: $\mathrm{PH}$ proc[s] é a pegada hídrica do passo "s" do processo (volume/tempo) e $\mathrm{P}[\mathrm{p}]$ a quantidade produzida do produto "p" (massa/tempo).

As pegadas hídricas podem ser associadas aos diversos passos do processo no sistema produtivo poderão ser totalmente atribuídas ao produto resultante (Hoekstra et al., 2011).

A partir disso, foi realizada entrevista via telefone, em julho de 2017, com quatro empresas (A, B, C e D) de beneficiamento de jeans localizadas na cidade de Caruaru, Pernambuco. Nesse questionário procurou identificar, com o responsável por cada empresa, os seguintes parâmetros: quantidade de peças produzidas por mês, volume total de água consumida no processo e a fonte de captação de água. Baseado nesses parâmetros foi possível elaborar o cálculo do valor estimado da pegada hídrica para as empresas estudadas.

\section{Resultados}

As empresas estudadas captam água no rio Ipojuca, em poço artesiano, ou utilizam caminhões pipa ou através da COMPESA. O consumo da água representa um desafio para as indústrias, visto que os recursos hídricos são um bem limitado.

As lavandarias em estudo apresentaram dados distintos, levando em consideração a demanda do mês de julho/2017. Esses dados podem ser visualizados na Tabela 1 .

As lavandarias " $\mathrm{A}$ " e "B" apresentaram uma demanda maior de peças. A lavandaria "A" consome 1.500.00 L.mês ${ }^{-1}$ com uma demanda de 84.540 peças por mês; a lavandaria "B" consome 1.260.000 L.mês ${ }^{-1}$, com uma demanda de 52.500 peças por mês. Ambas fazem reuso da água, porém deve-se ressaltar que segundo informações obtidas na entrevista, a lavandaria " $\mathrm{B}$ " consegue reutilizar cerca de $80 \%$ de sua água após tratamento em ETE. 
Tabela 1. Consumo de água nas indústrias investigadas. Fonte: Autores (2017).

\begin{tabular}{|c|c|c|c|c|c|}
\hline Lavandarias & Peças.dia-1 & Peça.mês'-1 & L.dia ${ }^{-1}$ & L.mês'-1 & $\mathbf{P H}$ \\
\hline $\mathrm{A}$ & 2.818 & 84.540 & 50.000 & 1.500 .000 & 17,74 \\
\hline B & 1.750 & 52.500 & 42.000 & 1.260 .000 & 24 \\
\hline $\mathrm{C}$ & 250 & 7.500 & 3.733 & 112.000 & 14,93 \\
\hline D & 300 & 9.000 & 20.000 & 600.000 & 60 \\
\hline
\end{tabular}

As indústrias "C" e "D" apresentaram uma menor demanda no mês de julho/2017. A indústria "C" consome 112.000 L.mês ${ }^{-1}$ com uma demanda de 7.500 peças por mês, e a " $D$ " consome 600.000 L.mês ${ }^{-1}$ com demanda de 9.000 peças por mês. Em estudo realizado no ITEP (2008 apud Marcelino, 2013), as lavandarias que, mensalmente, procedem a lavagem de, aproximadamente, 10.000 peças, estimou-se um consumo de água bruta de $20 \mathrm{~m}^{3} \cdot \mathrm{dia}^{-1}$ (Tabela 2).

Tabela 2. Consumo de água das lavandarias no pólo de confecções de Caruaru, PE. Fonte: adaptado ITEP (2008 apud Marcelino, 2013, p. 21). Autores devem preencher a tabela abaixo e deletar a sua.

\begin{tabular}{cc}
\hline $\begin{array}{c}\text { Quantidade de peças } \\
\text { por mês }\end{array}$ & $\begin{array}{c}\text { Consumo de água } \\
\text { bruta }\left(\mathbf{m}^{\mathbf{3}} \cdot \mathbf{d i a}^{-\mathbf{1}}\right)\end{array}$ \\
\hline $\begin{array}{c}\text { (>120.000) } \\
80.001 \text { a } 90.000\end{array}$ & 230 \\
70.001 a 80.000 & 170 \\
60.001 a 70.000 & 150 \\
50.001 a 60.000 & 130 \\
40.001 a 50.000 & 120 \\
30.001 a 40.000 & 100 \\
20.001 a 30.000 & 80 \\
10.001 a 20.000 & 60 \\
$<10.000$ & 40 \\
\hline
\end{tabular}

Baseado em Hoekstra et al. (2001, p. 21), "a pegada hídrica de um processo quando dividida pela quantidade de produto pode ser expressa como volume de água por unidade de produto". Conforme a equação descrita por Hoekstra et al. (2011), foi possível realizar a pegada hídrica do processo das indústrias, sendo assim realizado a divisão do consumo mensal de água pela quantidade de peça mensal e desta forma obtevese a quantidade de litros que cada peça consome nos processos de beneficiamento conforme tabela 1.
Fazendo um comparativo do valor da pegada hídrica dessas indústrias, verificou-se que apresentam características de empresa de pequeno porte, porém a indústria " $D$ " se destacou em relação às outras pelo consumo de água por peça, a mesma apresentou 60 L.peça ${ }^{-1}$, mesmo apresentando uma menor demanda o seu consumo é maior em relação às outras indústrias deste estudo. Destaca-se que a indústria "D" capta sua água do rio Ipojuca e a reutiliza em comparação com a indústria " $C$ " que não faz reuso da água no processo de beneficiamento do jeans.

Porém percebe-se mesmo a indústria " $D$ " reutilizar no seu processo, sua pegada ainda é superior as outras empresas estudadas.

A problemática ambiental das indústrias têxtil é o consumo e investimento em tecnologias para o reuso da água. Segundo a UNESCO (2017), sugere-se que os volumes das águas residuais industriais irão dobrar até 2025. Diante de tais situações podemos apontar o crescimento do uso dos recursos hídricos nestas atividades. O que poderá vir a causar alguns impactos como a escassez de água, a poluição dos rios entre outros. Conforme Lorena et al. (2016), estudos ambientais voltados para tratamento de efluentes e estabelecimento de indicadores de sustentabilidades é uma alternativa importante para redução dos poluentes gerados nas indústrias.

$\mathrm{O}$ processo de beneficiamento do jeans tem várias etapas e requer a utilização de itens como os corantes e outros aditivos, dessa forma foi possível constatar que cada etapa de beneficiamento irá gerar um resíduo (Quadro 1). Conforme Allègre et al. (2006), o setor têxtil utiliza cerca de 70 e 150 litros de água para $1 \mathrm{~kg}$ de peça seca durante $o$ processo de beneficiamento, considerando que uma peça jeans tem, em média, $500 \mathrm{~g}$.

Quadro 1. Etapas do processamento da Indústria Têxtil. Fonte: Adaptado do SINDITEXTIL (2017).

\begin{tabular}{|l|l|}
\hline Principais processos & Respectivas finalidades \\
\hline Alvejamento & Remover coloração amarela (natural) do material têxtil \\
\hline Tingimento & Conferir coloração ao material têxtil \\
\hline Secagem & Retirar umidade do material, através de energia térmica \\
\hline Amaciamento & Conferir toque agradável ao material \\
\hline Desengomagem & Remover a "goma" aplicada ao tecido \\
\hline
\end{tabular}


Amaciamento

Conferir toque agradável ao material

Gerador de Vapor (caldeira)

Fornecer vapor para os equipamentos e/ou operações que

envolvem transferência de calor

Atualmente, o crescimento da demanda comercial e a alteração do perfil do consumidor poderão vir a ser os maiores influenciadores no aumento de consumo de água nas indústrias têxtil. A UNESCO (2017) prevê que a demanda por água vai aumentar nas próximas décadas, principalmente nos setores agrícolas, nas indústrias e na produção de energia. Com isso podemos concluir que o aumento dos efluentes e rejeitos nos processos de produção também irá crescer. Conforme Barros (2012, p 213), "o estabelecimento de tecnologias disponíveis para reduzir a contaminação e extensão da contaminação não pode ser descartada". A partir deste pressuposto, o estabelecimento de estratégias sustentáveis para a redução do consumo e o reuso se tornam necessários, além de planejamento e operações corretas de reuso, que podem contribuir para a diminuição da poluição ambiental.

\section{Conclusão}

Diante do atual cenário de problemas ambientais associados aos conflitos socioeconômicos, é primordial a adoção de medidas efetivas quanto à gestão dos recursos hídricos, nesse contexto a pegada hídrica apresenta-se como um eficaz indicador de sustentabilidade.

A avaliação da pegada hídrica é uma ferramenta capaz de oferecer dados que auxiliam as pessoas a compreenderem de forma mais ampla a relação entre escassez e poluição da água, e as atividades e produtos.

No estudo realizado, a avaliação da pegada hídrica do processo de beneficiamento têxtil de uma lavandaria localizada no agreste de Pernambuco, mostrou que essa atividade é caracterizada como de significativo potencial poluidor devido ao elevado volume de água necessário para o seu processo produtivo.

$\mathrm{O}$ consumo de água nos processos industriais tende a ser crescente, em virtude do aumento populacional e da mudança do estilo de vida das pessoas, sendo fundamental que nos processos produtivos sejam implantadas intervenções concretas relativas à gestão da água, pois para que a crise hídrica possa ser controlada, tem de haver uma compatibilização entre a disponibilidade hídrica e as demandas atuais e futuras.

O estudo da pegada hídrica contribui para o entendimento do quantitativo de água que está sendo consumido no processo produtivo, para o caso de pegada hídrica em indústrias. A compreensão do consumo elevado e da escassez de água na sociedade pode funcionar como uma importante ferramenta na identificação de ações e medidas mitigadoras, como é o exemplo do reuso de água em algumas lavandarias de beneficiamento de jeans. Tais ações e medidas podem diminuir o consumo de água, beneficiando, não somente o meio ambiente, como também as indústrias, por reduzirem os custos com a compra ou a captação da água.

\section{Agradecimentos}

Os autores agradecem ao Programa de Pós-Graduação em Engenharia Ambiental da Universidade Federal Rural de Pernambuco.

\section{Referências}

ABIT. Associação Brasileira da Indústria Têxtil e de Confecções. $4^{\circ}$ Modavil leva informações sobre negócios da moda de Caruaru. Disponível em: http://www.abit.org.br. Acesso em 15 de agosto de 2017.

ALKAYA, E.; DEMIRER, G. N. 2014. Sustainable textile production: a case study from a woven fabric manufacturing mill in Turkey. Journal of Cleaner Production. V. 65, p. 595-603.

ALLÈGRE, C.; MOULIN, P.; MAISSEU, M.; CHARBIT, F. 2006. Treatment and reuse of reactive dyeing effluents. Journal of Membrane Science, v. 269, p. 15-34.

AMARAL, M. V. et. al. 2012. A Questão Ambiental no Pólo de Confecções de Caruaru: Um Primeiro Ensaio à Luz dos Instrumentos Econômicos de Proteção Ambiental. Estudos do CEPE, n. 35, p. 108-132.

ANA. 2016. Agência Nacional de Águas. Conjuntura dos Recursos Hídricos no Brasil.

BARBIERI, J. C. 2011. Gestão Ambiental Empresarial. $3^{a}$ ed. São Paulo: Saraiva.

BARROS, I. S. 2009. O Cenário do pólo de confecções do Agreste de Pernambuco. Recife. Anais do $2^{\circ}$ encontro de ensino pesquisa e extensão da faculdade Senac. 
BARROS, R. M. 2012. Tratado sobre resíduos sólidos: gestão, uso e sustentabilidade. Rio de Janeiro: Interciência, pp. 213.

BLENINGER, T.; KOTSUKA, L. K. 2015. Conceitos de Água Virtual e Pegada Hídrica: Estudo de Caso da Soja e Óleo de Soja no Brasil. Recursos Hídricos, v. 36, n. 1, p. 15-24.

BOSIRE, C. K. et al. 2015.Trends and spatial variation in water and land foot prints of meat and milk production systems in Kenya. Agriculture, Ecosystems \& Environment, v. 205, p. 36-47.

BUSS, M. V.; RIBEIRO, E. F.; SCHNEIDER, I. A. H.; MENEZES, J. C. S. S. 2015. Tratamento dos efluentes de uma lavandaria industrial: avaliação da capacidade de diferentes processos de tratamento. Revista de Engenharia Civil IMED, v. 2, n. 1, p. 2-10.

DONAIRE, D. 2011. Gestão Ambiental na empresa. $2^{\mathrm{a}}$ ed. São Paulo: Atlas.

ERCIN, A. E.; HOEKSTRA, A. Y. 2014. Water footprint scenarios for 2050: A global analysis. Environment International, v. 64, p. 71-82.

ERCIN, A. E.; MEKONNEN, M. M.; HOEKSTRA, A. Y. 2013. Sustainability of national consumption from a water resources perspective: the case study for France. Ecological Economics, v. 88, p. 133-147.

FAO. s.d. Water Scarcity. Disponível em: http://www.fao.org/land-water/water/waterscarcity/en/. Acesso em:9 de julho de 2017.

FARIA, F.; PACHECO, E. 2011. Experiências com Produção Mais Limpa no Setor Têxtil. SENAI. REDIGE - Revista de Design, Inovação e Gestão Estratégica. V. 2, n. 1, pp. 63 - 82. 2011.

FREY, K. 2000. Políticas públicas: um debate conceitual e reflexões referentes à prática da análise de políticas públicas no Brasil. Planejamento e Políticas Públicas. n. 21, p. 211259.

GALLI, A. A.; WIEDMANNB T.; ERCINC, E.; KNOBLAUCHD, D.; EWINGE, B.; GILJUMF, S. 2012. Integrating Ecological, Carbon and Water footprint into a "Footprint Family" of indicators: definition and role in tracking human pressure on the planet. Ecological Indicators, v. 16, p. 100-112.
GERBENS-LEENES, P. W. et al. 2012. Biofuel scenarios in a water perspective: The global blue and green water footprint of road transport in 2030. Global Environmental Change, v. 22, n. 3, p. 764-775.

HENKES, S. L. 2014. A política, o direito e o desenvolvimento: um estudo sobre a transposição do Rio São Francisco. Revista Direito GV. v. 10, n. 2, p. 497-534.

HOEKSTRA, A. Y., CHAPAGAIN, A. K., ALADAYA, M. M., MEKONNEM, M. M. 2011. The Water Footprint Assessment Manual - Setting the Global Standard. London: Earthscan, 224p.

HOEKSTRA, A. Y., HUNG, P. Q. 2003. Virtual water trade: a quantification of virtual water flows between nations in relation to international crop trade. Virtual Water Trade. Proceedings of the International Expert Meeting on Virtual Water Trade. Value of Water Research Report Series, v. 12, 120p.

HOEKSTRA, A. Y.; CHAPAGAIN, A. K.; ALADAYA, M. M. 2011. Manual de Avaliação da Pegada Hídrica - Estabelecendo o Padrão Global. Water Footprint Network, 216p.

LORENA, E. M. G. et al. 2016. Analysis of the procedural and wastewater treatment at a beverage bottling industry in the state of Pernambuco, Brazil. Revista Geama, v. 2, n. 4, p. 466-472.

MARCELINO, D. M. S. 2013. Avaliação da remoção de cor, matéria orgânica e sulfato de efluente têxtil através de reatores biológicos seqüenciais. 2013. 71f. Dissertação (Mestrado em Engenharia Civil e Ambiental) - Núcleo de Tecnologia do Centro Acadêmico do Agreste, Universidade Federal de Pernambuco, CaruaruPE.

MELO, M. C. S. 2012. Estudo de argamassas adicionadas de cinzas de algaroba geradas no arranjo produtivo local de confecções do agreste pernambucano. 121f. Dissertação (Mestrado em Engenharia Civil e Ambiental), Núcleo de Tecnologia do Centro Acadêmico do Agreste, Universidade Federal de Pernambuco, CaruaruPE.

MMA. 2003. Ministério do Meio Ambiente. Secretaria de Recursos Hídricos. Agência Nacional de Águas. Plano Nacional de Recursos Hídricos. Documento Base de Referência. Novembro. Brasília, 383p. 
MOTA, S. 2000. Introdução à engenharia ambiental. Rio de Janeiro. ABES, 416p.

OPA/CEPIS. 2002. Água: Não ao desperdício, não à escassez! Centro Panamericano de Engenharia Sanitária e Ciências do Ambiente. ABES. 50p.

PETERS, M. R. 2006. Potencialidade de uso de fontes alternativas de água para fins não potáveis em uma unidade residencial. 109f. Dissertação (Mestrado em Engenharia Ambiental), Universidade Federal de Santa Catarina, Santa Catarina.

SEBRAE. 2000. Serviço Brasileiro de Apoio a Micro e pequenas empresas: Eficiência da economia e a competitividade da cadeia têxtil brasileira, 2000. Disponível em: http://www.sebrae.com.br/br/cooperecrescer/cadei aprodutivas. Acesso em 15 de agosto 2017.

SILVA, V. P. R.; ALEIXO, D. O.; ALMEIDA, R. S. R.; CUNHA-CAMPOS, J. H. B.; ARAUJO, L. E. 2015. Modelo integrado das pegadas hídrica, ecológica e de carbono para o monitoramento da pressão humana sobre o planeta. Revista Ambiência, v. 11 n. 3 p. 639-649.

SILVA, V. P. R.; ALEIXO, D. O.; DANTAS, J.; MARACAJÁ, K. F. B.; ARAUJO, L. E. 2013. Uma medida de sustentabilidade ambiental: Pegada hídrica. Campina Grande-UAEA/UFCG. Revista Brasileira de Engenharia Agrícola e Ambiental, v. 17, n. 1, p. 100-105.

SINDITÊXTIL. 2012. Sindicato das Indústrias Têxteis do Estado de São Paulo. Têxtil e confecção: inovar, desenvolver e sustentar / Confederação Nacional da Indústria Associação Brasileira da Indústria Têxtil e de Confecção. Brasília: CNI/ABIT. Disponível em: http: Iwwww.sinditextilsp.org.br. Acesso em: 08 ago. 2017.

TEIXEIRA, M. J. M. 2012. Pegada hídrica: caso de estudo do tijolo cerâmico. 78f. Dissertação (Mestrado em Engenharia Civil) - Universidade de Trás-os-Montes e Alto Douro - Vila Real, Portugal.

TOMAZ, P. 2010. Aproveitamento de água de chuva: para áreas urbanas e fins não potáveis. São Paulo: editor Navegar. 486p.

TRINDADE, B., KATO, M.T., FLORENCIO, L., GAVAZZA, S. 2013. Effect of starch and ethanol as electron donors, and sulphate on the reductive decolourisation of azo dye Direct Black 22. 13th World Congress on Anaerobic Digestion. Santiago de Compostela. Spain.

UNESCO. 2017. United Nations Educational Scientific and Cultural Organization. Relatório Mundial das Nações Unidas sobre o Desenvolvimento dos Recursos Hídricos. Fatos e Números. 12p. Disponível em: http://unesdoc.unesco.org/images/0024/00247524 7552por.pdf. Acesso em: 10 jul. 2017.

WATER, U. N. 2006. Coping with Water Scarcity-A Strategic Issue and Priority for System-Wide Action. UN-Water: Geneva, Switzerland. 